\title{
A Suppository Chinese Medicine (Xilei-san) for Refractory Ulcerative Proctitis: A Pilot Clinical Trial
}

\author{
Ken Fukunaga $^{a}$ Nobuyuki Hida ${ }^{a}$ Kunio Ohnishi ${ }^{a}$ Yoshio Ohda ${ }^{a}$ Koji Yoshida ${ }^{a}$ \\ Takeshi Kusaka $^{a}$ Yoshio Jinno ${ }^{a, c}$ Kazuko Nagase $^{a}$ Shiro Nakamura ${ }^{a}$ \\ Muneo Kadobayashi ${ }^{d}$ Hiroto Miwab $^{b}$ Takayuki Matsumoto $^{a}$ \\ Divisions of a Lower Gastroenterology and ${ }^{\mathrm{b}}$ Upper Gastroenterology, Department of Internal Medicine, \\ ${ }^{c}$ Endoscopy Center, and ${ }^{d}$ Department of Pharmacy, Hyogo College of Medicine, Nishinomiya, Japan
}

Dear Sir,

Six patients with active ulcerative proctitis (UP) $($ male/female $=2 / 4)$ were treated with an originally developed suppository Xilei-san (SXS), a traditional Chinese medicine, which is composed of watermelon frost, calcite, cow gallstone, peal powder, borax, borneol, indigo, and ammonium chloride. This mixed traditional medicine has a long history in China and it has been accepted as a medicine for erosions and ulcerations of the tongue, pharynx and oral cavity. The therapeutic mechanism of XS has never been fully understood, but some domestic reports inform that ulcerative colitis (UC) has been pro- posed as a possible indication of peroral XS. We have hypothesized that XS might be a useful therapeutic option for active UP patients refractory to conventional topical therapies if it would be applied in suppository form. A prospective open-labeled clinical trial was held from April 1, 2005 to December 31, 2006 to evaluate the efficacy of SXS for active refractory UP patients.

Mean $( \pm$ SD) age and duration of UP of the enrolled patients were $36.2 \pm 9.1$ years and $56.3 \pm 47.4$ months, respectively. All patients had an episode of treatment with a combination of either peroral 5- aminosalicylic acid (5-ASA) or sulphasalazine (SASP) together with conventional suppositories. Eligible patients had a confirmed diagnosis of UP in clinical and endoscopically active relapse although current suppositories had been given for $>4$ weeks. According to the previous study [1], entry was restricted to patients with disease not extending $15 \mathrm{~cm}$ beyond the anus. Patients having any episode of drug allergy and pregnant or lactating women were excluded. SXS was manufactured originally in the pharmacology division of our institute. Our prototype SXS contained 0.1 $\mathrm{g} /$ piece of Xilei-San powder (Beijing Tong
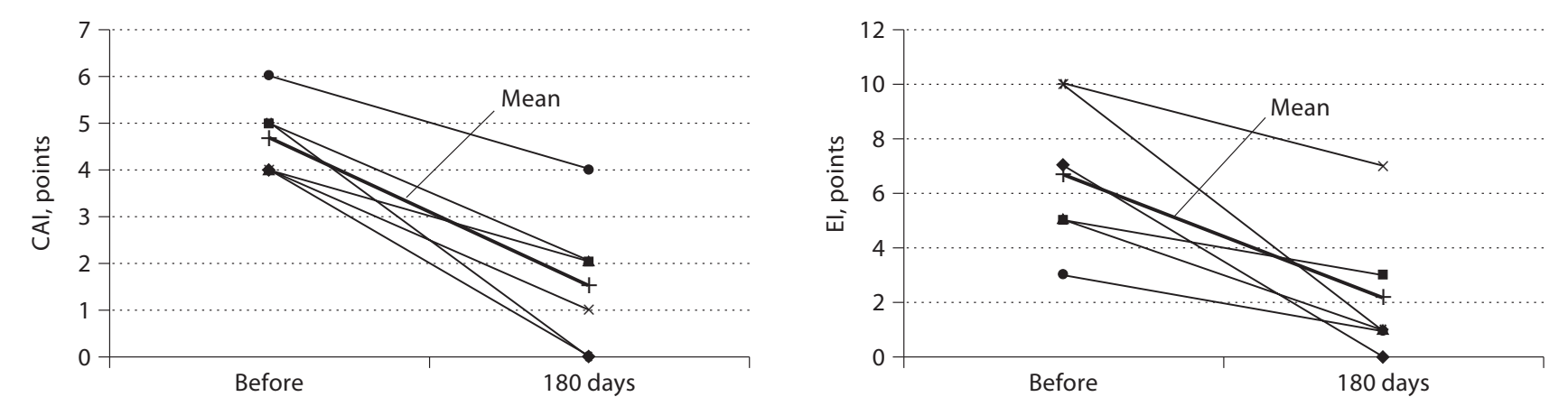

Fig. 1. CAI and EI scores were compared before and after the trial. Significant improvements of the mean CAI $(4.67 \pm 0.82$ vs. $1.50 \pm 1.52 ; \mathrm{p}<0.03)$ and $\mathrm{EI}(6.67 \pm 2.88$ vs. $2.17 \pm 2.56 ; \mathrm{p}<0.03)$ were proven between before and after the trial.

\section{KARGER}

Fax +41613061234

E-Mail karger@karger.ch

www.karger.com (c) 2007 S. Karger AG, Basel $0012-2823 / 07 / 0753-0146 \$ 23.50 / 0$

Accessible online at: www.karger.com/dig
Ken Fukunaga, MD, PhD

Division of Lower Gastroenterology

Department of Internal Medicine, Hyogo College of Medicine, Mukogawa

Nishinomiya Hyogo, 663-8501 (Japan)

Tel./Fax+81 79845 6662, E-Mail kenfukunaga1@r4.dion.ne.jp 
Ren Tang Ltd., Co., Beijing, China) mixed with commercially available carrier glyceride. SXS was given 1 piece/day for 180 days after obtaining sufficient informed consent, and after the local ethics committee had approved a human protocol for this trial. The combined peroral administration was allowed to continue in the same dosage if it had been started $>4$ weeks before the enrollment. Any combined topical therapy except SXS was never allowed after starting the trial. Clinical and endoscopic efficiencies of SXS were evaluated before and 180 days after beginning the trial utilizing the Rachmilewitz clinical activity index (CAI) and the endoscopic index (EI) [2]. Also, we compared the quality-of-life (QoL) of the patients before and after the trial using an IBDQ [3]. Statistical analysis was made using the Wilcoxon signed-rank test. $\mathrm{p}<0.05$ was considered statistically significant. Patients tolerated the trial well, and there were no cases either presenting specific adverse reactions or stopping due to deterioration. Their macroscopic bloody feces disappeared in $49.2 \pm 25.6$ days (range 21-85). Significant improvements of the mean CAI and EI were proven after the trial (fig.1). Also, the mean IBDQ score was improved significantly after the trial $(168.8 \pm 19.7$ vs. $191.0 \pm 12.5 ; \mathrm{p}<0.03)$.

Suppository is a suitable approach for UP patients. Topical treatment with sulphasalazine (SASP), 5-aminosalicylic acid (5-ASA; the active moiety of SASP), and hydrocortisone has demonstrated its efficiency for active UP patients when they are formulated as suppositories [4-6]. However, some patients have revealed a strong resistance to these current topical therapies because of insufficient clinical improvements and/or adverse side effects. SXS might have the potential to be an adjunct therapeutic option for UP patients with such past episode(s). Fragmental facts have recognized that XS has the potential to have an effect in UC not only in China, but also in Japan [7]. Ohu et al. [8] have proven that XS maintained the gastric mucosal blood flow in chemically induced gastric ulcer models in mice. In this way of thinking, active UP patients should be the best candidates for SXS because it takes effect directly at the disease focus. Clinical symptoms seen in active UP are sometimes similar to UC patients with more expanded colitis. Symptoms such as frequent bloody diarrhea, abdominal pain, and weight loss and consequently chronic recurrent inflammation within the recto-sigmoidal mucosa might initiate serious damage to their QoL. In conclusion, we report here the results of our pilot clinical trial of a suppository-formed Chinese traditional medicine, Xilei-san, for active UP patients. SXS was found to suppress the prevailing inflammation during the 180-day trial with a striking effect on both CAI and EI. Further studies in large cohorts of patients are warranted to evaluate its therapeutic effect and mechanism, and a randomized, controlled, doubleblind placebo study has been commenced based upon the results obtained from this pilot trial.

\section{References}

1 d'Albasio G, Paoluzi P, Camieri M, et al: Maintenance treatment of ulcerative proctitis with mesalazine suppositories: a doubleblind placebo-controlled trial. The Italian IBD Study Group. Am J Gastroenterol 1998; 93:799-803.

2 Rachmilewitz D: Coated mesalazine (5-aminosalicylic acid) versus sulphasalazine in the treatment of active ulcerative colitis: a randomised trial. BMJ 1989;298:82-86.

3 Irvine EJ: A quality-of-life index for inflammatory bowel disease. Can J Gastroenterol 1993;7:155-159.

4 Williams CN: Role of rectal formulations: suppositories. Scand J Gastroenterol Suppul 1990;172:60-62.

5 Campieri M, Gionchetti P, Belluzzi A, et al: 5-Aminosalicylic acid suppositories in the management of ulcerative colitis. Dis Colon Rectum 1989;32:398-399.

6 Farup PG, Hovde O, Halvorsen FA, Raknerud N, Brodin U: Mesalazine suppositories versus hydrocortisone foam in patients with distal ulcerative colitis: a comparison of the efficacy and practicality of two topical treatment regimens. Scand J Gastroenterol 1995; 30:164-170.

7 Amano K, Amano K, Kume K, Nakamura K, Chin S: Xileisan as the new drug for the treatment of ulcerative colitis. Annual Report of the Research Committee of Inflammatory Bowel Disease (in Japanese). Tokyo, Ministry of Health, Labor and Welfare of Japan, 2000, pp 137-138.

8 Ohu B, Higuchi K, Fukuda T, et al: Anti-ulceration efficiency of Xilei San (Chinese medicine) (in Japanese). Prog Med 1989;9: 2847-2852. 